\title{
Termolecular one-pot synthesis of symmetrical azines of 4-acetyl-3-arylsydnones. Hydrazone and azine derivatives of 4-acetyl-3-arylsydnones, their spectral characterization and biological properties
}

\author{
Prashant S. Shinge, Shanta G. Mallur and Bharati V. Badami* \\ P.G. Department of Chemistry, Karnatak University, Dharwad-580 003, India \\ E-mail : bbadami@rediffimail.com \\ Manuscript received 9 June 2004, revised 18 December 2004, accepted 22 March 2005
}

\begin{abstract}
Hydrazones of 4-acetyl-3-arylsydnones (2a-o) were prepared by heating 4-acetyl-3-arylsydnones (1a-o) with excess of hydrazine hydrate and the corresponding symmetrical azines (3a-o) were obtained by a termolecular reaction of 4-acetyl-3arylsydnones with hydrazine hydrate $(2: 1$ ratio). Hydrazones and azines were screened for their antimicrobial activity and few of these compounds exhibit inhibitory action more than the reference drugs used.
\end{abstract}

The synthesis, biological and pharmacological studies of a large number of sydnone derivatives have been reported from our laboratory ${ }^{1-3}$. In continuation of synthetic work on sydnones, we now report the synthesis of some hydrazones of 4-acetyl-3-arylsydnones ${ }^{4}$ and termolecular one-step synthesis of the corresponding symmetrical azines by varying the stoichiometric quantity of 4-acetyl-3arylsydnones (1a-o) and the reagent hydrazine hydrate.

\section{Results and discussion}

The hydrazones (2a-o) were obtained by treating the 4acetyl-3-arylsydnones (1a-o) with excess of hydrazine hydrate. These hydrazones were resistant to further reaction with aldehyde/ketone and also with another molecule of 4acetyl-3-arylsydnone, even after prolonged heating and the corresponding azines could not be prepared. The low reactivity of the $-\mathrm{NH}_{2}$ group of the hydrazones may be attributed to the electron-withdrawing property of the sydnone ring which is a mesoionic dipolar heterocycle (Figs. 1 and 2). Hence, we thought of carrying out a termolecular reaction with two moles of 4-acetyl-3-arylsydnones (1a-o) and one mole of hydrazine hydrate, since hydrazine hydrate is a symmetrical binucleophile it can readily react simultaneously with two carbonyl carbons. The azines (3a-o) were obtained in $\sim 85 \%$ yield in about 10 minutes (Scheme 1).

The IR spectra of all the hydrazones (2a-0) showed bands at $3424 \mathrm{~cm}^{-1}$ and $3388 \mathrm{~cm}^{-1}$ for asymmetric and symmetric $v_{\mathrm{NH}_{2}}$ stretching. The bands at $1727 \mathrm{~cm}^{-1}$ and 1641 $\mathrm{cm}^{-1}$ are due to $\mathrm{v}_{\mathrm{C}=\mathrm{O}}$ of the sydnone ring and $\mathrm{v}_{\mathrm{C}=\mathrm{N}}$ respectively. The ${ }^{1} \mathrm{H}$ NMR (300 MHz) (DMSO- $\left.d_{6}\right)$ spectra of these

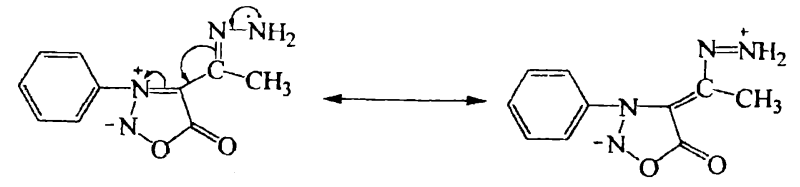

Fig. 1

Fig. 2

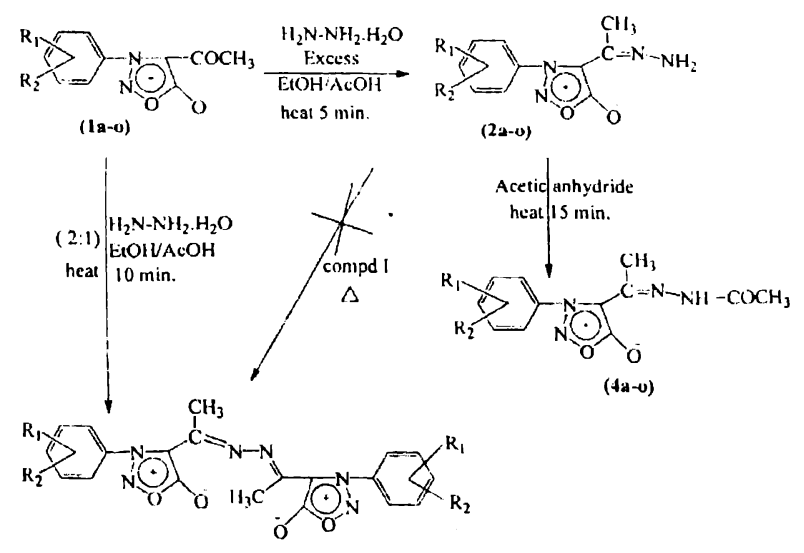

(3a-0)

$$
\begin{array}{rlll}
\text { 1-4 a, } \mathrm{R}_{1}=\mathrm{H}, & \mathrm{R}_{2}=\mathrm{H} & \text { h, } \mathrm{R}_{1}=\mathrm{H}, & \mathrm{R}_{2}=4-\mathrm{Br} \\
\text { b, } \mathrm{R}_{1}=\mathrm{H}, & \mathrm{R}_{2}=4-\mathrm{CH}_{3} & \text { i, } \mathrm{R}_{1}=\mathrm{H}, & \mathrm{R}_{2}=4-\mathrm{CO}_{2} \mathrm{CH}_{3} \\
\text { c, } \mathrm{R}_{1}=\mathrm{H}, & \mathrm{R}_{2}=2-\mathrm{CH}_{3} & \text { j, } \mathrm{R}_{1}=\mathrm{H}, & \mathrm{R}_{2}=4-\mathrm{CO}_{2} \mathrm{C}_{2} \mathrm{H}_{5} \\
\text { d, } \mathrm{R}_{1}=\mathrm{H}, & \mathrm{R}_{2}=4-\mathrm{OCH}_{3} & \text { k, } \mathrm{R}_{1}=3-\mathrm{CH}_{3}, & \mathrm{R}_{2}=4-\mathrm{CH}_{3} \\
\text { e, } \mathrm{R}_{1}=\mathrm{H}, & \mathrm{R}_{2}=2-\mathrm{OCH}_{3} & \text { l, } \mathrm{R}_{1}=2-\mathrm{CH}_{3}, & \mathrm{R}_{2}=5-\mathrm{CH}_{3} \\
\text { f, } \mathrm{R}_{1}=\mathrm{H}, & \mathrm{R}_{2}=4-\mathrm{Cl} & \text { m, } \mathrm{R}_{1}=4-\mathrm{CH}_{3}, & \mathrm{R}_{2}=3-\mathrm{Cl} \\
\text { g, } \mathrm{R}_{1}=\mathrm{H}, & \mathrm{R}_{2}=3-\mathrm{Cl} & \text { n, } \mathrm{R}_{1}=2-\mathrm{OCH}_{3}, & \mathrm{R}_{2}=4-\mathrm{Cl} \\
& & \text { o, } \mathrm{R}_{1}=4-\mathrm{Cl} . & \mathrm{R}_{2}=3-\mathrm{F}
\end{array}
$$

Scheme I 
compounds showed a singlet at $\delta 2.37(3 \mathrm{H})$ for the methyl protons of the hydrazone group, and another broad peak at $\delta 10.40(2 \mathrm{H})$ for amino group $\left(\mathrm{D}_{2} \mathrm{O}\right.$ exchanged). The aromatic protons appeared in the region $\delta 6.40-7.00$. The IR spectra of the azines (3a-o) exhibited band at $1741 \mathrm{~cm}^{-1}$ and $1642 \mathrm{~cm}^{-1}$ due to $v_{\mathrm{C}=\mathrm{O}}$ of the sydnone ring and $v_{\mathrm{C}=\mathrm{N}}$. The absence of the $-\mathrm{NH}_{2}$ band confirms the formation of azine. The ${ }^{1} \mathrm{H}$ NMR (DMSO- $d_{6}$ ) resonance showed magnetic equivalence for the protons of one half of the molecule with the other. All these compounds showed a singlet at $\delta 3.9(6 \mathrm{H})$ corresponding to the methyl protons on the azine group and the aromatic protons appeared at $\delta 7.00$ 7.30. The formation of the azine was confirmed by the EIMass spectrum of a typical compound the $p$-chloro substituted derivative (3f). The molecular ion at $\mathrm{m} / \mathrm{z} 472$ was not observed but the fragment at $m / z 414$, accounts for the loss of $-\mathrm{NO}$ and $-\mathrm{CO}$ from the sydnone ring. This fragmentation is typical of the sydnone ring. The IR spectra of the ar etyl derivatives of the hydrazones (4a-0) showed a broad band at $3300 \mathrm{~cm}^{-1}$ due to $v_{\mathrm{NH}}$ stretching and $1758 \mathrm{crn}^{-1}$ due to $v_{\mathrm{C}=\mathrm{O}}$ of the sydnone ring, while the amide $\mathrm{v}_{\mathrm{C}=\mathrm{O}}$ appeared at $1659 \mathrm{~cm}^{-1}$. ${ }^{1} \mathrm{HNMR}$ (DMSO- $d_{6}$ ) spectra showed a singlet at $\delta 2.60(3 \mathrm{H})$ for the methyl protons of $-\mathrm{COCH}_{3}$ and $\delta$ $2.20(3 \mathrm{H})$ of hydrazone group. The - $\mathrm{NH}$ proton appeared at $\delta 10.3\left(1 \mathrm{H}, \mathrm{D}_{2} \mathrm{O}\right.$ exchanged $)$ and the aromatic protons were observed in the region of $\delta 7.41-7.38$.

\section{Antimicrobial activity :}

The preliminary antimicrobial testing has been carried out by cup-plate method. The antimicrobial activity was done against two pathogenic bacteria Escherichia coli and Pseudomonas pyocyanous and Asperigillus niger and Rhizocona bataticola as the fungal strains. The reference drugs used were Norfloxacin and Griseofulvin respectively. Compounds ( $2 \mathbf{f}, \mathbf{g}, \mathbf{h}, \mathbf{m}, \mathbf{n}$ and $\mathbf{o}$ ) with halogen substitutions on the phenyl ring exhibited antibacterial activity greater than the reference drug against both the bacteria, while only methyl $(\mathbf{2 b}, \mathbf{c}, \mathbf{k}$ and $\mathbf{l})$ group substitution increased the activity against both the fungi. The halogen substituted azines (3f, $\mathbf{g}$ and $\mathbf{h}$ ) showed enhanced antibacterial activity against both the organisms compared to norfloxacin. Only the $m-\mathrm{Cl}(\mathbf{3 g})$ derivative was found to be more active against $R$. bataticoa while all the other compounds were moderately active. Amongst the acetyl derivatives (4a-o) only one derivative viz. 4-Cl-3-F (4o) exhibited activity greater than the reference drug against $E$. coli while all the other compounds were moderately active against both the bacteria. The 4-bromo (4h) and 4-carbethoxy (4j) substitutions enhanced the antifungal activity against both the fungi, while other derivatives were moderately active (Tables 1-3).
Table 1. Antimicrobial activity of compounds (2a-o)

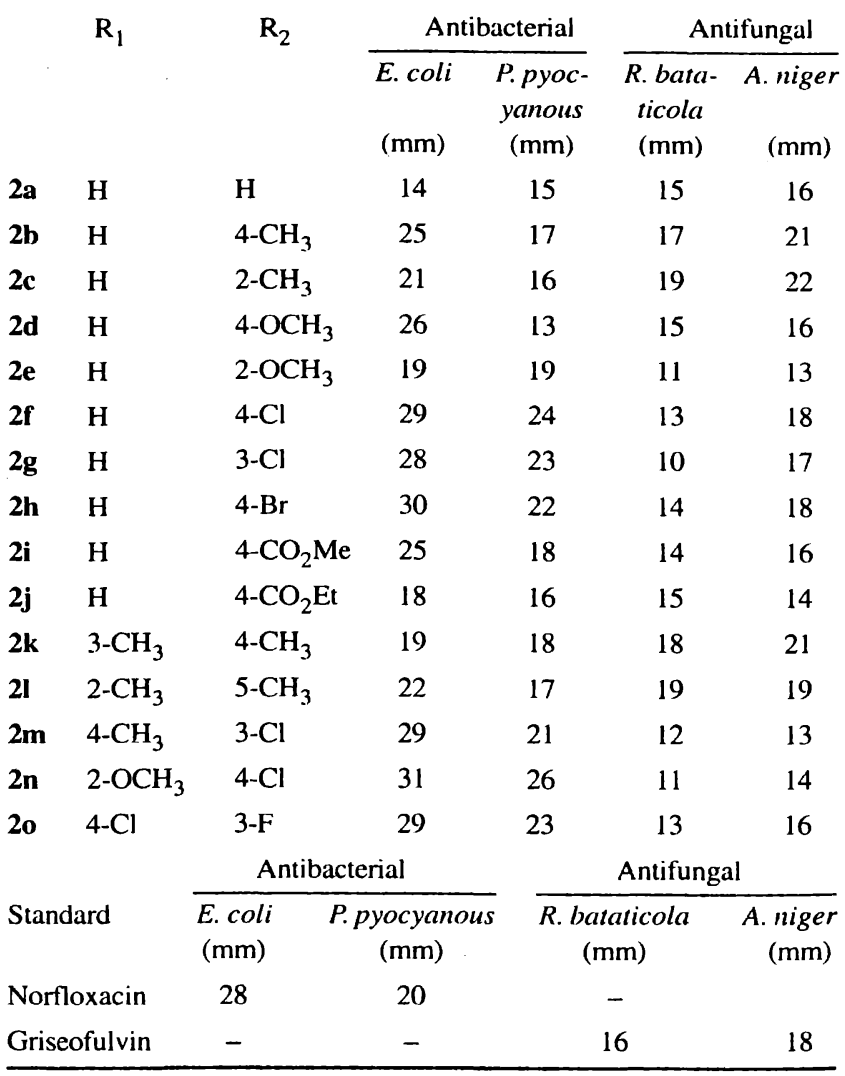

\section{Experimental}

Melting points were determined in open capillary tube and are uncorrected. The IR spectra were recorded on Nicolet Impact-410 FT-IR spectrophotometer, using $\mathrm{KBr}$ pellets. ${ }^{1} \mathrm{H}$ NMR was recorded on Brucker Varian- $300 \mathrm{MHz}$ FT-NMR in DMSO- $d_{6}$ with TMS as an internal standard. Mass spectra were recorded on a MI Ver. 14 on UIC 002002. Elemental analysis was carried out using Heraus $\mathrm{CHN}$ rapid analyzer.

Synthesis of hydrazones of 4-acetyl-3-aryl sydnones (2ao) : 4-Acetyl-3-aryl sydnones $(0.005 \mathrm{~mol})$ were dissolved in ethanol (4 ml) containing 3-4 drops of acetic acid. Hydrazine hydrate $(0.025 \mathrm{~mol})$ was added and the reaction mixture warmed on a water-bath for $2 \mathrm{~min}$. The reaction mixture was then diluted with water till turbidity was observed and warmed on water-bath for another $5 \mathrm{~min}$, cooled and filtered. The bright orange crystalline compounds obtained were recrystallised from methanol. 2a (85\%), m.p. 169-170 (Found : C, 55.00: H, 4.60; N, 25.60. $\mathrm{C}_{10} \mathrm{H}_{10} \mathrm{~N}_{4} \mathrm{O}_{2}$ calcd. for : $\mathrm{C}, 55.03 ; \mathrm{H}, 4.02 ; \mathrm{N}, 25.62 \%$ ); $v_{\max } 3424$ and $3388\left(\mathrm{NH}_{2}\right), 1727$ (sydnone $\mathrm{C}=\mathrm{O}$ ), $1641(\mathrm{C}=\mathrm{N}) ; \delta_{\mathrm{H}}\left(\mathrm{DMSO}-d_{6}\right) 10.40(2 \mathrm{H}$, br, exchanged with 
Table 2. Antimicrobial activity of compounds (3a-o)

\begin{tabular}{|c|c|c|c|c|c|c|}
\hline \multirow{2}{*}{ F } & \multirow[t]{2}{*}{$\mathrm{R}_{1}$} & \multirow[t]{2}{*}{$\mathbf{R}_{2}$} & \multicolumn{2}{|c|}{ Antibacterial } & \multicolumn{2}{|c|}{ Antifungal } \\
\hline & & & $\begin{array}{l}\text { E. coli } \\
(\mathrm{mm})\end{array}$ & $\begin{array}{l}\text { P. pyoc- } \\
\text { yanous } \\
(\mathrm{mm})\end{array}$ & $\begin{array}{c}\text { R. bata- } \\
\text { ticola } \\
(\mathrm{mm})\end{array}$ & $\begin{array}{c}\text { A. niger } \\
(\mathrm{mm})\end{array}$ \\
\hline $3 \mathbf{a}$ & $\mathbf{H}$ & $\mathrm{H}$ & 14 & 15 & 15 & 16 \\
\hline $3 \mathbf{b}$ & $\mathbf{H}$ & 4- $\mathrm{CH}_{3}$ & 25 & 17 & 13 & 16 \\
\hline $3 c$ & $\mathrm{H}$ & $2-\mathrm{CH}_{3}$ & 21 & 16 & 14 & 15 \\
\hline 3d & $\mathbf{H}$ & $4-\mathrm{OCH}_{3}$ & 26 & 13 & 15 & 16 \\
\hline $3 \mathbf{e}$ & $\mathbf{H}$ & $2-\mathrm{OCH}_{3}$ & 19 & 19 & 11 & 13 \\
\hline $3 f$ & $\mathbf{H}$ & $4-\mathrm{Cl}$ & 30 & 23 & 13 & 17 \\
\hline $3 \mathrm{~g}$ & $\mathrm{H}$ & $3-\mathrm{Cl}$ & 29 & 25 & 19 & 15 \\
\hline $3 \mathbf{h}$ & $\mathbf{H}$ & $4-\mathrm{Br}$ & 31 & 24 & 14 & 13 \\
\hline $3 \mathbf{i}$ & $\mathbf{H}$ & 4- $\mathrm{CO}_{2} \mathrm{Me}$ & 25 & 18 & 14 & 16 \\
\hline $3 \mathbf{j}$ & $\mathbf{H}$ & $4-\mathrm{CO}_{2} \mathrm{Et}$ & 18 & 16 & 15 & 14 \\
\hline $3 \mathbf{k}$ & $3-\mathrm{CH}_{3}$ & 4- $\mathrm{CH}_{3}$ & 19 & 18 & 15 & 17 \\
\hline 31 & $2-\mathrm{CH}_{3}$ & $5-\mathrm{CH}_{3}$ & 22 & 17 & 13 & 13 \\
\hline $3 \mathbf{m}$ & 4- $\mathrm{CH}_{3}$ & $3-\mathrm{Cl}$ & 25 & 13 & 12 & 13 \\
\hline $3 n$ & $2-\mathrm{OCH}_{3}$ & $4-\mathrm{Cl}$ & 18 & 16 & 11 & 14 \\
\hline \multirow[t]{2}{*}{30} & $4-\mathrm{Cl}$ & $3-F$ & 21 & 15 & 13 & 16 \\
\hline & & \multicolumn{2}{|c|}{ Antibacterial } & \multicolumn{3}{|c|}{ Antifungal } \\
\hline \multicolumn{2}{|c|}{ Standard } & $\begin{array}{l}\bar{E} . \text { coli } \\
(\mathrm{mm})\end{array}$ & $\begin{array}{l}\text { pyocyanous } \\
\text { (mm) }\end{array}$ & $\begin{array}{r}\text { R.bat } \\
\text { (m }\end{array}$ & $\begin{array}{l}\text { taticola } \\
\mathrm{nm})\end{array}$ & $\begin{array}{c}\text { A. niger } \\
(\mathrm{mm})\end{array}$ \\
\hline \multicolumn{2}{|c|}{ Norfloxacin } & 28 & 20 & & - & - \\
\hline \multicolumn{2}{|c|}{ Griseofulvin } & - & - & & 16 & 18 \\
\hline
\end{tabular}

$\left.\mathrm{D}_{2} \mathrm{O}, \mathrm{NH}_{2}\right), 6.5-7.41(5 \mathrm{H}, \mathrm{m}, \mathrm{Ar}-\mathrm{H}), 2.0\left(3 \mathrm{H}, \mathrm{s}, \mathrm{CH}_{3}\right) ; \mathrm{m} / z$ $218\left(\mathrm{M}^{+}\right)$; $2 \mathrm{~b}(84 \%)$, m.p.171-172 ${ }^{\circ}$ (Found : C, 56.80; H, 520; $\mathrm{N} 24.10 . \mathrm{C}_{11} \mathrm{H}_{12} \mathrm{~N}_{4} \mathrm{O}_{2}$ calcd. for : $\mathrm{C}, 56.87 ; \mathrm{H}, 5.21$; $\mathrm{N}, 24.13 \%) ; v_{\max } 3422$ and $3380\left(\mathrm{NH}_{2}\right), 1730$ (sydnone $\mathrm{C}=\mathrm{O}), 1643(\mathrm{C}=\mathrm{N}) ; \delta_{\mathrm{H}}\left(\mathrm{DMSO}-d_{\sigma}\right) 10.42(2 \mathrm{H}, \mathrm{br}$, exchanged with $\left.\mathrm{D}_{2} \mathrm{O}, \mathrm{NH}_{2}\right), 6.40(2 \mathrm{H}, \mathrm{d}, \mathrm{Ar}-\mathrm{H}), 7.00(2 \mathrm{H}, \mathrm{d}$, Ar-H), $2.0\left(3 \mathrm{H}, \mathrm{s}, \mathrm{CH}_{3}\right), 2.3\left(3 \mathrm{H}, \mathrm{s}, \mathrm{CH}_{3}\right) ; \delta_{\mathrm{C}}\left(\mathrm{DMSO}-d_{6}\right)$ 21.8 (q, Ar- $\left.\mathrm{CH}_{3}\right), 28.4$ (q, $\left.\mathrm{CH}_{3}\right), 124$ (q, $\left.\mathrm{Ar}-\mathrm{CH}_{3}\right), 129.2$ (q, Ar-sydnone), $130.3(\mathrm{q}, \mathrm{C}=\mathrm{N}), 143.3$ (q, sydnone ring $\mathrm{C}$ ), 166.6 (a, sydnone $\mathrm{C}=\mathrm{O}$ ), 125.1 and 130.2 (2C each Ar ring); $\mathrm{m} / \mathrm{z} 232\left(\mathrm{M}^{+}\right)$; 2c (80\%), m.p.119-120 (Found : C, 56.80; $\mathrm{H}, 520 ; \mathrm{N}, 24.10 . \mathrm{C}_{11} \mathrm{H}_{12} \mathrm{~N}_{4} \mathrm{O}_{2}$ calcd. for : C, 56.87; $\mathrm{H}$, $5.21 ; \mathrm{N}, 24.13 \%) ; \mathrm{v}_{\max } 3421$ and $3385\left(\mathrm{NH}_{2}\right), 1732$ (sydnone $\mathrm{C}=\mathrm{O}), 1640(\mathrm{C}=\mathrm{N}) ; \delta_{\mathrm{H}}\left(\mathrm{DMSO}-d_{6}\right) 10.41(2 \mathrm{H}, \mathrm{br}$, exchanged with $\left.\mathrm{D}_{2} \mathrm{O}, \mathrm{NH}_{2}\right), 6.40(1 \mathrm{H}, \mathrm{d}, \mathrm{Ar}-\mathrm{H}), 7.20(1 \mathrm{H}$, d, Ar-H), $7.15(2 \mathrm{H}, \mathrm{m}, \mathrm{Ar}-\mathrm{H}), 2.1\left(3 \mathrm{H}, \mathrm{s}, \mathrm{CH}_{3}\right) 2.4(3 \mathrm{H}, \mathrm{s}$, $\left.\mathrm{CH}_{3}\right) ; m / z 232\left(\mathrm{M}^{+}\right) ; 2 \mathrm{~d}(78 \%)$, m.p. 174-176 (Found : C, 53.20; $\mathrm{H}, 4.80 ; \mathrm{N}, 22.60 . \mathrm{C}_{11} \mathrm{H}_{12} \mathrm{~N}_{4} \mathrm{O}_{3}$ calcd. for : C, 53.21; $\mathrm{H}, 4.87 ; \mathrm{N}, 22.58 \%) ; v_{\max } 3422$ and $3380\left(\mathrm{NH}_{2}\right), 1730$ (sydnone $\mathrm{C}=\mathrm{O}), 1643(\mathrm{C}=\mathrm{N}) ; \delta_{\mathrm{H}}\left(\mathrm{DMSO}-d_{6}\right) 10.42(2 \mathrm{H}$, br, exchanged with $\left.\mathrm{D}_{2} \mathrm{O}, \mathrm{NH}_{2}\right), 6.40(2 \mathrm{H}, \mathrm{d}, \mathrm{Ar}-\mathrm{H}), 7.00$ ( $2 \mathrm{H}, \mathrm{d}, \mathrm{Ar}-\mathrm{H}), 2.0\left(3 \mathrm{H}, \mathrm{s}, \mathrm{CH}_{3}\right), 3.8\left(3 \mathrm{H}, \mathrm{s}, \mathrm{CH}_{3}\right) ; \mathrm{m} / \mathrm{z} 248$
Table 3. Antimicrobial activity of compounds (4a-o)

\begin{tabular}{|c|c|c|c|c|c|c|}
\hline & \multirow[t]{2}{*}{$\mathrm{R}_{1}$} & \multirow[t]{2}{*}{$\mathbf{R}_{2}$} & \multicolumn{2}{|c|}{ Antibacterial } & \multicolumn{2}{|c|}{ Antifungal } \\
\hline & & & $\begin{array}{l}\text { E. coli } \\
(\mathrm{mm})\end{array}$ & $\begin{array}{l}\text { P. pyoc- } \\
\text { yanous } \\
(\mathrm{mm})\end{array}$ & $\begin{array}{c}\text { R. bata- } \\
\text { ticola } \\
(\mathrm{mm})\end{array}$ & $\begin{array}{c}\text { A. niger } \\
\text { (mm) }\end{array}$ \\
\hline $4 \mathbf{a}$ & $\mathbf{H}$ & $\mathrm{H}$ & 14 & 15 & 15 & 16 \\
\hline $4 \mathbf{b}$ & $\mathbf{H}$ & $4-\mathrm{CH}_{3}$ & 25 & 17 & 13 & 16 \\
\hline $4 c$ & $\mathbf{H}$ & $2-\mathrm{CH}_{3}$ & 21 & 16 & 15 & 17 \\
\hline $4 d$ & $\mathrm{H}$ & $4-\mathrm{OCH}_{3}$ & 25 & 13 & 15 & 16 \\
\hline $4 \mathbf{e}$ & $\mathrm{H}$ & $2-\mathrm{OCH}_{3}$ & 19 & 18 & 11 & 13 \\
\hline $4 f$ & $\mathrm{H}$ & 4-Cl & 24 & 17 & 13 & 15 \\
\hline $4 \mathrm{~g}$ & $\mathrm{H}$ & $3-\mathrm{Cl}$ & 23 & 16 & 10 & 17 \\
\hline $4 h$ & $\mathrm{H}$ & $4-\mathrm{Br}$ & 26 & 15 & 19 & 21 \\
\hline $4 i$ & $\mathbf{H}$ & $4-\mathrm{CO}_{2} \mathrm{Me}$ & 25 & 18 & 14 & 16 \\
\hline $4 j$ & $\mathbf{H}$ & 4- $\mathrm{CO}_{2} \mathrm{Et}$ & 18 & 16 & 21 & 22 \\
\hline $4 k$ & $3-\mathrm{CH}_{3}$ & 4- $\mathrm{CH}_{3}$ & 19 & 18 & 16 & 15 \\
\hline 41 & $2-\mathrm{CH}_{3}$ & $5-\mathrm{CH}_{3}$ & 22 & 17 & 15 & 16 \\
\hline $4 m$ & 4- $\mathrm{CH}_{3}$ & $3-\mathrm{Cl}$ & 24 & 16 & 12 & 13 \\
\hline $4 n$ & $2-\mathrm{OCH}_{3}$ & $4-\mathrm{Cl}$ & 25 & 18 & 11 & 14 \\
\hline 40 & \multirow[t]{2}{*}{$4-\mathrm{Cl}$} & $3-\mathrm{F}$ & 32 & 15 & 13 & 16 \\
\hline & & \multicolumn{2}{|c|}{ Antibacterial } & \multicolumn{3}{|c|}{ Antifungal } \\
\hline \multicolumn{2}{|c|}{ Standard } & $\begin{array}{l}\text { E. coli } \\
(\mathrm{mm})\end{array}$ & $\begin{array}{c}\text { pyocyanous } \\
(\mathrm{mm})\end{array}$ & R. $b a$ & $\begin{array}{l}\text { taticola } \\
\mathrm{nm})\end{array}$ & $\begin{array}{c}\text { A. niger } \\
(\mathrm{mm})\end{array}$ \\
\hline \multicolumn{2}{|c|}{ Norfloxacin } & 28 & 20 & & - & - \\
\hline \multicolumn{2}{|c|}{ Griseofulvin } & - & - & & 16 & 18 \\
\hline
\end{tabular}

$\left(\mathrm{M}^{+}\right) ; 2 \mathrm{e}(75 \%)$, m.p. $160-161^{\circ}$ (Found : C, 53.21; H, 4.79; $\mathrm{N}, 22.63 . \mathrm{C}_{11} \mathrm{H}_{12} \mathrm{~N}_{4} \mathrm{O}_{3}$ calcd. for : $\mathrm{C}, 53.21 ; \mathrm{H}, 4.87 ; \mathrm{N}$, $22.58 \%$ ); $v_{\max } 3420$ and $3385\left(\mathrm{NH}_{2}\right), 1735$ (sydnone $\mathrm{C}=\mathrm{O}$ ), $1641(\mathrm{C}=\mathrm{N}) ; \delta_{\mathrm{H}}\left(\mathrm{DMSO}-d_{6}\right) 10.42(2 \mathrm{H}, \mathrm{br}$, exchanged with $\left.\mathrm{D}_{2} \mathrm{O}, \mathrm{NH}_{2}\right), 6.43(1 \mathrm{H}, \mathrm{d}, \mathrm{Ar}-\mathrm{H}), 7.35(1 \mathrm{H}, \mathrm{d}, \mathrm{Ar}-\mathrm{H}), 7.15$ $(2 \mathrm{H}, \mathrm{m}, \mathrm{Ar}-\mathrm{H}), 2.1\left(3 \mathrm{H}, \mathrm{s}, \mathrm{CH}_{3}\right), 3.8\left(3 \mathrm{H}, \mathrm{s}, \mathrm{CH}_{3}\right) ; \mathrm{m} / z 248$ $\left(\mathrm{M}^{+}\right)$; $2 \mathrm{f}(85 \%)$, m.p.192-193\% (Found : C, 47.60; H, 3.60; $\mathrm{N}, 22.18 . \mathrm{C}_{10} \mathrm{H}_{9} \mathrm{ClN}_{4} \mathrm{O}_{2}$ calcd. for : $\mathrm{C}, 47.61 ; \mathrm{H}, 3.60 ; \mathrm{N}$, $22.22 \%$ ); $v_{\max } 3422$ and $3380\left(\mathrm{NH}_{2}\right), 1727$ (sydnone $\mathrm{C}=\mathrm{O}$ ), $1643(\mathrm{C}=\mathrm{N}) ; \delta_{\mathrm{H}}\left(\mathrm{DMSO}-d_{6}\right) 10.42(2 \mathrm{H}, \mathrm{br}$, exchanged with $\left.\mathrm{D}_{2} \mathrm{O}, \mathrm{NH}_{2}\right), 6.40(2 \mathrm{H}, \mathrm{d}, \mathrm{Ar}-\mathrm{H}), 7.00(2 \mathrm{H}, \mathrm{d}, \mathrm{Ar}-\mathrm{H}), 2.0$ $\left(3 \mathrm{H}, \mathrm{s}, \mathrm{CH}_{3}\right) ; m / z 252\left(\mathrm{M}^{+}\right) ; 2 \mathrm{~g}(88 \%)$, m.p.145-146 (Found : $\mathrm{C}, 47.59 ; \mathrm{H}, 3.59 ; \mathrm{N}, 22.23 . \mathrm{C}_{10} \mathrm{H}_{9} \mathrm{ClN}_{4} \mathrm{O}_{2}$ calcd. for : $\mathrm{C}$, 47.61; H, 3.60; N, 22.22\%); $v_{\max } 3420$ and $3385\left(\mathrm{NH}_{2}\right)$, 1735 (sydnone $\mathrm{C}=\mathrm{O}), 1641(\mathrm{C}=\mathrm{N}) ; \delta_{\mathrm{H}}\left(\mathrm{DMSO}-d_{6}\right) 10.39$ ( $2 \mathrm{H}$, br, exchanged with $\left.\mathrm{D}_{2} \mathrm{O}, \mathrm{NH}_{2}\right), 6.41(1 \mathrm{H}, \mathrm{d}, \mathrm{Ar}-\mathrm{H}), 7.30$ $(1 \mathrm{H}, \mathrm{d}, \mathrm{Ar}-\mathrm{H}), 7.15(1 \mathrm{H}, \mathrm{m}, \mathrm{Ar}-\mathrm{H}), 7.8(1 \mathrm{H}, \mathrm{s}, \mathrm{Ar}-\mathrm{H}), 2.1(3 \mathrm{H}$, s, $\left.\mathrm{CH}_{3}\right) ; m / 2252\left(\mathrm{M}^{+}\right) ; 2 \mathrm{~h}(82 \%)$, m.p.146-147\% (Found : C, 40.50; H, 3.02; N, 18.90. $\mathrm{C}_{10} \mathrm{H}_{9} \mathrm{BrN}_{4} \mathrm{O}_{2}$ calcd. for : $\mathrm{C}$, 40.54; $\mathrm{H}, 3.06 ; \mathrm{N}, 18.92 \%) ; \mathrm{v}_{\max } 3420$ and $3378\left(\mathrm{NH}_{2}\right)$, 1728 (sydnone $\mathrm{C}=\mathrm{O}), 1642(\mathrm{C}=\mathrm{N}) ; \delta_{\mathrm{H}}\left(\mathrm{DMSO}-d_{6}\right) 10.43$ $\left(2 \mathrm{H}\right.$, br, exchanged with $\left.\mathrm{D}_{2} \mathrm{O}, \mathrm{NH}_{2}\right), 6.43(2 \mathrm{H}, \mathrm{d}, \mathrm{Ar}-\mathrm{H})$, 


\section{J. Indian Chem. Soc., Vol. 82, July 2005}

$7.32(2 \mathrm{H} \mathrm{d}, \mathrm{Ar}-\mathrm{H}), 2.2\left(3 \mathrm{H}, \mathrm{s}, \mathrm{CH}_{3}\right) ; \mathrm{m} / 2297\left(\mathrm{M}^{+}\right) ; 2 \mathrm{i}(70 \%)$, m.p.128-129 (Found : C, 52.10; H, 4.40; N, 20.28. $\mathrm{C}_{12} \mathrm{H}_{12} \mathrm{~N}_{4} \mathrm{O}_{4}$ calcd. for : C, 52.17; H, 4.38; N, 20.28\%); $v_{\max } 3422$ and $3380\left(\mathrm{NH}_{2}\right), 1742$ (ester $\left.\mathrm{C}=\mathrm{O}\right), 1727$ (sydnone $\mathrm{C}=\mathrm{O}), 1643(\mathrm{C}=\mathrm{N}) ; \delta_{\mathrm{H}}\left(\mathrm{DMSO}-d_{6}\right) 10.42(2 \mathrm{H}$, br, exchanged with $\left.\mathrm{D}_{2} \mathrm{O}, \mathrm{NH}_{2}\right), 6.87(2 \mathrm{H}, \mathrm{d}, \mathrm{Ar}-\mathrm{H}), 7.45$ $(2 \mathrm{H}, \mathrm{d}, \mathrm{Ar}-\mathrm{H}), 2.1\left(3 \mathrm{H}, \mathrm{s}, \mathrm{CH}_{3}\right), 3.8\left(3 \mathrm{H}, \mathrm{s}, \mathrm{CH}_{3}\right) ; \mathrm{m} / z 276$ $\left(\mathrm{M}^{+}\right)$; $2 \mathrm{j}\left(73 \%\right.$ ), m.p. $111-112^{\circ}$ (Found : C, 53.80; H, 4.80; $\mathrm{N}, 19.20 . \mathrm{C}_{13} \mathrm{H}_{14} \mathrm{~N}_{4} \mathrm{O}_{4}$ calcd. for : $\mathrm{C}, 53.78 ; \mathrm{H}, 4.86 ; \mathrm{N}$, $19.30 \%) ; v_{\max } 3422$ and $3380\left(\mathrm{NH}_{2}\right), 1732$ (sydnone $\mathrm{C}=\mathrm{O}$ ), $1745($ ester $\mathrm{C}=\mathrm{O}), 1643(\mathrm{C}=\mathrm{N}) ; \delta_{\mathrm{H}}\left(\mathrm{DMSO}-d_{6}\right) 10.42(2 \mathrm{H}$, br, exchanged with $\left.\mathrm{D}_{2} \mathrm{O}, \mathrm{NH}_{2}\right), 6.87(2 \mathrm{H}, \mathrm{d}, \mathrm{Ar}-\mathrm{H}), 7.45$ $(\mathrm{H}, \mathrm{d}, \mathrm{Ar}-\mathrm{H}), 2.1\left(3 \mathrm{H}, \mathrm{s}, \mathrm{CH}_{3}\right), 3.8\left(2 \mathrm{H}, \mathrm{q}, \mathrm{CH}_{2}\right), 1.8(3 \mathrm{H}, \mathrm{t}$, $\left.\mathrm{CH}_{3}\right) ; \mathrm{m} / z 290\left(\mathrm{M}^{+}\right) ; 2 \mathbf{2 k}(86 \%)$, m.p. 159-160 (Found : C, 55.50; H, 5.70; N, 22.70. $\mathrm{C}_{12} \mathrm{H}_{14} \mathrm{~N}_{4} \mathrm{O}_{2}$ calcd. for : C, 58.51; $\mathrm{H}, 5.73 ; \mathrm{N}, 22.76 \%) ; v_{\max } 3422$ and $3380\left(\mathrm{NH}_{2}\right), 1732$ $(\mathrm{C}=\mathrm{O}), 1643(\mathrm{C}=\mathrm{N}) ; \delta_{\mathrm{H}}\left(\mathrm{DMSO}-d_{6}\right) 10.42(2 \mathrm{H}$, br, exchanged with $\left.\mathrm{D}_{2} \mathrm{O}, \mathrm{NH}_{2}\right), 6.45(1 \mathrm{H}, \mathrm{d}, \mathrm{Ar}-\mathrm{H}), 7.21(1 \mathrm{H}$, d, Ar-H), $7.5(1 \mathrm{H}, \mathrm{s}, \mathrm{Ar}-\mathrm{H}), 2.1\left(3 \mathrm{H}, \mathrm{s}, \mathrm{CH}_{3}\right), 2.0(3 \mathrm{H}, \mathrm{s}$, $\left.\mathrm{CH}_{3}\right), 1.9\left(3 \mathrm{H}, \mathrm{s}, \mathrm{CH}_{3}\right) ; \mathrm{m} / z 246\left(\mathrm{M}^{+}\right) ; 2 \mathrm{l}(68 \%)$, m.p. 128 $129^{\circ}$ (Found : C, 55.50; H, 5.70; N, 22.70. $\mathrm{C}_{12} \mathrm{H}_{14} \mathrm{~N}_{4} \mathrm{O}_{2}$ calcd. for : C, 58.51; H, 5.73; N, 22.76\%); $v_{\max } 3421$ and $3375\left(\mathrm{NH}_{2}\right), 1732$ (sydnone $\left.\mathrm{C}=\mathrm{O}\right), 1643(\mathrm{C}=\mathrm{N}) ; \delta_{\mathrm{H}}(\mathrm{DMSO}-$ $\left.d_{6}\right) 10.41\left(2 \mathrm{H}\right.$, br, exchanged with $\left.\mathrm{D}_{2} \mathrm{O}, \mathrm{NH}_{2}\right), 6.43(1 \mathrm{H}, \mathrm{d}$, Ar-H), $7.18(1 \mathrm{H}, \mathrm{d}, \mathrm{Ar}-\mathrm{H}), 7.4(1 \mathrm{H}, \mathrm{s}, \operatorname{Ar}-\mathrm{H}), 2.1(3 \mathrm{H}, \mathrm{s}$, $\left.\mathrm{CH}_{3}\right), 2.0\left(3 \mathrm{H}, \mathrm{s}, \mathrm{CH}_{3}\right), 1.9\left(3 \mathrm{H}, \mathrm{s}, \mathrm{CH}_{3}\right) ; \mathrm{m} / \mathrm{z} 246\left(\mathrm{M}^{+}\right) ; 2 \mathrm{~m}$ (80\%), m.p. $131-132^{\circ}$ (Found : C, 49.60; H, 4.70; N, 21.00 . $\mathrm{C}_{11} \mathrm{H}_{11} \mathrm{ClN}_{4} \mathrm{O}_{2}$ calcd. for : $\mathrm{C}, 49.60 ; \mathrm{H}, 4.71 ; \mathrm{N}, 21.05 \%$ ); $v_{\text {max }} 3420$ and $3375\left(\mathrm{NH}_{2}\right), 1730$ (sydnone $\mathrm{C}=\mathrm{O}$ ), 1643 $(\mathrm{C}=\mathrm{N}) ; \delta_{\mathrm{H}}\left(\mathrm{DMSO}-d_{6}\right) 10.43\left(2 \mathrm{H}\right.$, br, exchanged with $\mathrm{D}_{2} \mathrm{O}$, $\left.\mathrm{NH}_{2}\right), 6.41(1 \mathrm{H}, \mathrm{d}, \mathrm{Ar}-\mathrm{H}), 7.16(1 \mathrm{H}, \mathrm{d}, \mathrm{Ar}-\mathrm{H}), 7.5(1 \mathrm{H}, \mathrm{s}$, $\mathrm{Ar}-\mathrm{H}), 2.1\left(3 \mathrm{H}, \mathrm{s}, \mathrm{CH}_{3}\right), 2.0\left(3 \mathrm{H}, \mathrm{s}, \mathrm{CH}_{3}\right) ; \mathrm{m} / z 266\left(\mathrm{M}^{+}\right) ; 2 \mathrm{n}$ (60\%), m.p. 147-148 (Found : C, 46.79; H, 3.90; N, 19.80. $\mathrm{C}_{11} \mathrm{H}_{11} \mathrm{ClN}_{4} \mathrm{O}_{3}$ calcd. for : $\mathrm{C}, 46.80 ; \mathrm{H}, 3.93 ; \mathrm{N}, 19.86 \%$ ); $v_{\max } 3420$ and $3375\left(\mathrm{NH}_{2}\right), 1730$ (sydnone $\mathrm{C}=\mathrm{O}$ ), 1643 $(\mathrm{C}=\mathrm{N}) ; \delta_{\mathrm{H}}\left(\mathrm{DMSO}-d_{6}\right) 10.43\left(2 \mathrm{H}, \mathrm{br}\right.$, exchanged with $\mathrm{D}_{2} \mathrm{O}$, $\left.\mathrm{NH}_{2}\right), 6.41(\mathrm{lH}, \mathrm{d}, \mathrm{Ar}-\mathrm{H}), 7.16(1 \mathrm{H}, \mathrm{d}, \mathrm{Ar}-\mathrm{H}), 7.5(1 \mathrm{H}, \mathrm{s}$, $\mathrm{Ar}-\mathrm{H}), 2.1\left(3 \mathrm{H}, \mathrm{s}, \mathrm{CH}_{3}\right), 3.8\left(3 \mathrm{H}, \mathrm{s}, \mathrm{CH}_{3}\right) ; \mathrm{m} / z 282\left(\mathrm{M}^{+}\right) ; 2 \mathrm{o}$ (78\%), m.p. 131-133 ${ }^{\circ}$ (Found : C, 44.40; H, 2.90; N, 20.70. $\mathrm{C}_{10} \mathrm{H}_{8} \mathrm{ClFN}_{4} \mathrm{O}_{2}$ calcd. for : C, 44.44; $\mathrm{H}, 2.99 ; \mathrm{N}, 20.74 \%$ ); $v_{\max } 3420$ and $3375\left(\mathrm{NH}_{2}\right), 1738$ (sydnone $\mathrm{C}=\mathrm{O}$ ), 1643 $(\mathrm{C}=\mathrm{N}) ; \delta_{\mathrm{H}}\left(\mathrm{DMSO}-d_{6}\right) 10.45\left(2 \mathrm{H}\right.$, br, exchanged with $\mathrm{D}_{2} \mathrm{O}$, $\left.\mathrm{NH}_{2}\right), 6.48(1 \mathrm{H}, \mathrm{d}, \mathrm{Ar}-\mathrm{H}), 7.24(1 \mathrm{H}, \mathrm{d}, \mathrm{Ar}-\mathrm{H}), 7.6(1 \mathrm{H}, \mathrm{s}$, $\mathrm{Ar}-\mathrm{H}), 2.1\left(3 \mathrm{H}, \mathrm{s}, \mathrm{CH}_{3}\right) ; m / z 270\left(\mathrm{M}^{+}\right)$.

Synthesis of azines of 4-acetyl-3-arylsydnones (3a-0) : 4-Acetyl-3-arylsydnones $(0.01 \mathrm{~mol})$ were dissolved in ethanol ( $4 \mathrm{ml}$ ) containing 3-4 drops of acetic acid. Hydrazine hydrate $(0.005 \mathrm{~mol})$ was then added and the reaction mixture was warmed on a water-bath for $10 \mathrm{~min}$, cooled and the solid separated was filtered. White crystalline com- pounds were obtained after crystallisation from methanol to give (3a-o). 3a (85\%), m.p. 220-22 $1^{\circ}$ (Found : C, 59.40; $\mathrm{H}, 3.90 ; \mathrm{N}, 20.70 . \mathrm{C}_{20} \mathrm{H}_{16} \mathrm{~N}_{6} \mathrm{O}_{4}$ calcd. for : C, 59.39; $\mathrm{H}$, $3.98 ; \mathrm{N}, 20.78 \%$ ); $v_{\max } 1741$ (sydnone $\left.\mathrm{C}=\mathrm{O}\right), 1624(\mathrm{C}=\mathrm{N})$; $\delta_{\mathrm{H}}\left(\mathrm{DMSO}-d_{6}\right) 7.0-7.30(10 \mathrm{H}, \mathrm{m}, \mathrm{Ar}-\mathrm{H}), 3.9\left(6 \mathrm{H}, \mathrm{s}, 2 \mathrm{CH}_{3}\right)$; $\mathrm{m} / \mathrm{z} 404\left(\mathrm{M}^{+}\right)$; 3b (88\%), m.p. 236-237\% (Found : C. 61.08; $\mathrm{H}, 4.60 ; \mathrm{N}, 19.41 . \mathrm{C}_{22} \mathrm{H}_{20} \mathrm{~N}_{6} \mathrm{O}_{4}$ calcd. for : C, 61.10: $\mathrm{H}$, $4.66 ; \mathrm{N}, 19.43 \%) ; v_{\max } 1739$ (sydnone $\left.\mathrm{C}=\mathrm{O}\right) .1620(\mathrm{C}=\mathrm{N})$; $\delta_{\mathrm{H}}\left(\mathrm{DMSO}-d_{6}\right) 6.80(4 \mathrm{H}, \mathrm{d}, \mathrm{Ar}-\mathrm{H}), 7.20(4 \mathrm{H} . \mathrm{d}, \mathrm{Ar}-\mathrm{H}), 3.9$ $\left(6 \mathrm{H}, \mathrm{s}, 2 \mathrm{CH}_{3}\right), 2.3\left(6 \mathrm{H}, \mathrm{s}, 2 \mathrm{CH}_{3}\right) ; \delta_{\mathrm{C}}\left(\mathrm{DMSO}-d_{6}\right) 22.0(2 \mathrm{C}$, $\left.\mathrm{Ar}-\mathrm{CH}_{3}\right), 33.5\left(2 \mathrm{C}, \mathrm{CH}_{3}\right), 124.5$ (q, 2C, $\left.\mathrm{Ar}-\mathrm{CH}_{3}\right), 131.2$ (q, 2C, Ar-sydnone), 140.1 (q, 2C, C=N), 150.2 (q, 2C, sydnone ring), 168.8 (q, 2C, $\mathrm{C}=\mathrm{O}$ ), 126.0 and 132.3 (4C each, $\mathrm{Ar}$ ); $\mathrm{m} / \mathrm{z} 432\left(\mathrm{M}^{+}\right)$; 3c (72\%) m.p. 210-211 ${ }^{\circ}$ (Found : C, 61.08; $\mathrm{H}, 4.63 ; \mathrm{N}, 19.40 . \mathrm{C}_{22} \mathrm{H}_{20} \mathrm{~N}_{6} \mathrm{O}_{4}$ calcd. for : $\mathrm{C}, 61.10 ; \mathrm{H}$. $4.66 ; \mathrm{N}, 19.43 \%) ; v_{\max } 1740$ (sydnone $\left.\mathrm{C}=\mathrm{O}\right), 1614(\mathrm{C}=\mathrm{N})$; $\delta_{\mathrm{H}}\left(\mathrm{DMSO}-d_{6}\right) 6.40(2 \mathrm{H}, \mathrm{d}, \mathrm{Ar}-\mathrm{H}), 7.20(2 \mathrm{H}, \mathrm{d}, \mathrm{Ar}-\mathrm{H}), 7.15$ $(4 \mathrm{H}, \mathrm{m}, \mathrm{Ar}-\mathrm{H}), 3.8\left(3 \mathrm{H}, \mathrm{s}, 2 \mathrm{CH}_{3}\right), 2.4\left(3 \mathrm{H}, \mathrm{s}, 2 \mathrm{CH}_{3}\right) ; \mathrm{m} / \mathrm{z}$ $432\left(\mathrm{M}^{+}\right) ; 3 \mathbf{d}(82 \%)$, m.p. 229-230 (Found : C, 56.86; H, 4.32; N. 18.08. $\mathrm{C}_{22} \mathrm{H}_{20} \mathrm{~N}_{6} \mathrm{O}_{6}$ calcd. for : C, 56.89; H, 4.34; $\mathrm{N}, 18.10 \%) ; v_{\max } 1740($ sydnone $\mathrm{C}=\mathrm{O}), 1613(\mathrm{C}=\mathrm{N}) ; \delta_{\mathrm{H}}$ (DMSO- $\left.d_{6}\right) 6.73(4 \mathrm{H}, \mathrm{d}, \mathrm{Ar}-\mathrm{H}), 7.52(4 \mathrm{H}, \mathrm{d}, \mathrm{Ar}-\mathrm{H}), 3.9(6 \mathrm{H}$, s, $\left.2 \mathrm{CH}_{3}\right), 3.8\left(6 \mathrm{H}, \mathrm{s}, 2 \mathrm{CH}_{3}\right) ; \mathrm{m} / \mathrm{z} 464\left(\mathrm{M}^{+}\right) ; 3 \mathrm{e}(80 \%)$, m.p. $211-212^{\circ}$ (Found: C, 56.86; H, 4.32; N, 18.08. $\mathrm{C}_{22} \mathrm{H}_{20} \mathrm{~N}_{6} \mathrm{O}_{6}$ calcd. for : C, 56.89; H, 4.34; N, 18.10\%); $v_{\max } 1735$ (sydnone $\mathrm{C}=\mathrm{O}), 1611(\mathrm{C}=\mathrm{N}) ; \delta_{\mathrm{H}}\left(\mathrm{DMSO}-d_{6}\right) 6.43(2 \mathrm{H}, \mathrm{d}$, Ar-H), 7.35 (2H, d, Ar-H), 7.15 (4H, m, Ar-H), 3.9 (6H, s, $\left.2 \mathrm{CH}_{3}\right), 3.8\left(6 \mathrm{H}, \mathrm{s}, 2 \mathrm{CH}_{3}\right) ; \mathrm{m} / z 464\left(\mathrm{M}^{+}\right) ; 3 \mathrm{f}(90 \%)$, m.p. 240-241 ${ }^{\circ}$ (Found : C, 50.74; H, 2.95; N, 17.74. $\mathrm{C}_{20} \mathrm{H}_{14} \mathrm{Cl}_{2} \mathrm{~N}_{6} \mathrm{O}_{4}$ calcd. for : C, 50.76; $\mathrm{H}, 2.98 ; \mathrm{N}, 17.76 \%$ ); $v_{\max } 1745$ (sydnone $\left.\mathrm{C}=\mathrm{O}\right), 1613(\mathrm{C}=\mathrm{N}) ; \delta_{\mathrm{H}}\left(\mathrm{DMSO}-d_{6}\right)$ $6.45(4 \mathrm{H}, \mathrm{d}, \mathrm{Ar}-\mathrm{H}), 7.05$ (4H, d, Ar-H), $3.7\left(6 \mathrm{H}, \mathrm{s}, 2 \mathrm{CH}_{3}\right)$; $\mathrm{m} / \mathrm{z} 472\left(\mathrm{M}^{+}\right) ; 3 \mathrm{~g}(90 \%)$, m.p. 209-210 (Found : C, 50.74; $\mathrm{H}, 2.95 ; \mathrm{N}, 17.74 . \mathrm{C}_{20} \mathrm{H}_{14} \mathrm{Cl}_{2} \mathrm{~N}_{6} \mathrm{O}_{4}$ calcd. for : $\mathrm{C}, 50.76 ; \mathrm{H}$, 2.98; N, 17.76\%); $v_{\max } 1745$ (sydnone $\mathrm{C}=\mathrm{O}$ ), $1611(\mathrm{C}=\mathrm{N}$ ); $\delta_{\mathrm{H}}\left(\mathrm{DMSO}-d_{6}\right) 6.41(2 \mathrm{H}, \mathrm{d}, \mathrm{Ar}-\mathrm{H}), 7.30(2 \mathrm{H}, \mathrm{d}, \mathrm{Ar}-\mathrm{H}), 7.15$ $(2 \mathrm{H}, \mathrm{m}, \mathrm{Ar}-\mathrm{H}), 7.8(2 \mathrm{H}, \mathrm{s}, \mathrm{Ar}-\mathrm{H}), 3.9\left(6 \mathrm{H}, \mathrm{s}, 2 \mathrm{CH}_{3}\right) ; \mathrm{m} / \mathrm{z}$ $472\left(\mathrm{M}^{+}\right)$; 3h (85\%), m.p. 213-214 ${ }^{\circ}$ (Found : C, 42.70; H, 2.50; N, 14.93. $\mathrm{C}_{20} \mathrm{H}_{14} \mathrm{Br}_{2} \mathrm{~N}_{6} \mathrm{O}_{4}$ calcd. for : $\mathrm{C}, 42.73 ; \mathrm{H}$, $2.51 ; \mathrm{N}, 14.95 \%) ; v_{\max } 1748$ (sydnone $\left.\mathrm{C}=\mathrm{O}\right), 1612(\mathrm{C}=\mathrm{N})$; $\delta_{\mathrm{H}}\left(\mathrm{DMSO}-d_{6}\right) 6.43(4 \mathrm{H}, \mathrm{d}, \mathrm{Ar}-\mathrm{H}), 7.32(4 \mathrm{H}, \mathrm{d}, \mathrm{Ar}-\mathrm{H}), 3.9$ $\left(6 \mathrm{H}, \mathrm{s}, 2 \mathrm{CH}_{3}\right) ; \mathrm{m} / \mathrm{z} 562\left(\mathrm{M}^{+}\right) ; 3 \mathbf{i}(80 \%)$, m.p. $213-214^{\circ}$ (Found : C, 55.36; H, 3.84; N, 16.13. $\mathrm{C}_{24} \mathrm{H}_{20} \mathrm{~N}_{6} \mathrm{O}_{8}$ calcd. for: $\mathrm{C}, 55.39 ; \mathrm{H}, 3.87 ; \mathrm{N}, 16.15 \%$ ); $v_{\max } 1740$ (sydnone $\mathrm{C}=\mathrm{O}), 1745($ ester $\mathrm{C}=\mathrm{O}), 1613(\mathrm{C}=\mathrm{N}) ; \delta_{\mathrm{H}}\left(\right.$ DMSO- $\left.d_{6}\right) 6.87$ $(4 \mathrm{H}, \mathrm{d}, \mathrm{Ar}-\mathrm{H}), 7.45(4 \mathrm{H}, \mathrm{d}, \mathrm{Ar}-\mathrm{H}), 3.88\left(6 \mathrm{H}, \mathrm{s}, 2 \mathrm{CH}_{3}\right), 3.8$ $\left(6 \mathrm{H}, \mathrm{s}, 2 \mathrm{CH}_{3}\right) ; \mathrm{m} / \mathrm{z} 520\left(\mathrm{M}^{+}\right) ; 3 \mathbf{j}(82 \%)$, m.p. $227-228^{\circ}$ (Found : C, 56.89; H, 4.40; N, 15.29. $\mathrm{C}_{26} \mathrm{H}_{24} \mathrm{~N}_{6} \mathrm{O}_{8}$ calcd. for : C, 56.93; H, 4.41; N, 15.32\%); $v_{\max } 1739$ (sydnone $\mathrm{C}=\mathrm{O}), 1745($ ester $\mathrm{C}=\mathrm{O}), 1613(\mathrm{C}=\mathrm{N}) ; \delta_{\mathrm{H}}\left(\mathrm{DMSO}-d_{6}\right) 6.87$ 
(4H, d, Ar- $\mathrm{H}), 7.45(4 \mathrm{H}, \mathrm{d}, \mathrm{Ar}-\mathrm{H}), 3.9\left(6 \mathrm{H}, \mathrm{s}, 2 \mathrm{CH}_{3}\right), 3.8$ $\left(4 \mathrm{H}, \mathrm{q}, 2 \mathrm{CH}_{2}\right), 1.8\left(6 \mathrm{H}, \mathrm{t}, 2 \mathrm{CH}_{3}\right) ; \mathrm{m} / z 548\left(\mathrm{M}^{+}\right) ; 3 \mathrm{k}(70 \%)$, m.p. 209-210 (Found: C, 62.56; H, 5.22; N, 18.23. $\mathrm{C}_{24} \mathrm{H}_{24} \mathrm{~N}_{6} \mathrm{O}_{4}$ calcd. for : C, 62.60; H, 5.25; N, 18.25\%); $v_{\max } 1732$ (sydnone $\left.\mathrm{C}=\mathrm{O}\right), 1643(\mathrm{C}=\mathrm{N}) ; \delta_{\mathrm{H}}\left(\mathrm{DMSO}-d_{6}\right)$ $6.45(2 \mathrm{H}, \mathrm{d}, \mathrm{Ar}-\mathrm{H}), 7.21(2 \mathrm{H}, \mathrm{d}, \mathrm{Ar}-\mathrm{H}), 7.5(2 \mathrm{H}, \mathrm{s}, \mathrm{Ar}-\mathrm{H})$, $3.74\left(6 \mathrm{H}, \mathrm{s}, 2 \mathrm{CH}_{3}\right), 2.0\left(6 \mathrm{H}, \mathrm{s}, 2 \mathrm{CH}_{3}\right), 1.9\left(6 \mathrm{H}, \mathrm{s}, 2 \mathrm{CH}_{3}\right)$; $\mathrm{m} / \mathrm{z} 460\left(\mathrm{M}^{+}\right)$; 31 (68\%), m.p. 214-215 (Found : C, 62.56; $\mathrm{H}, 5.22 ; \mathrm{N}, 18.23 . \mathrm{C}_{24} \mathrm{H}_{24} \mathrm{~N}_{6} \mathrm{O}_{4}$ calcd. for : C, 62.60; $\mathrm{H}$, $5.25 ; \mathrm{N}, 18.25 \%) ; v_{\max } 1742$ (sydnone $\left.\mathrm{C}=\mathrm{O}\right), 1613(\mathrm{C}=\mathrm{N})$; $\delta_{\mathrm{H}}\left(\mathrm{DMSO}-d_{6}\right) 6.43(2 \mathrm{H}, \mathrm{d}, \mathrm{Ar}-\mathrm{H}), 7.18(2 \mathrm{H}, \mathrm{d}, \mathrm{Ar}-\mathrm{H}), 7.4$ $(2 \mathrm{H}, \mathrm{s}, \mathrm{Ar}-\mathrm{H}), 3.9\left(6 \mathrm{H}, \mathrm{s}, 2 \mathrm{CH}_{3}\right), 2.0\left(6 \mathrm{H}, \mathrm{s}, 2 \mathrm{CH}_{3}\right), 1.9$ $\left(6 \mathrm{H}, \mathrm{s}, 2 \mathrm{CH}_{3}\right) ; \mathrm{m} / \mathrm{z} 460\left(\mathrm{M}^{+}\right) ; 3 \mathrm{~m}(80 \%)$, m.p. $219-220^{\circ}$ (Found: C, 52.70; $\mathrm{H}, 3.60 ; \mathrm{N}, 16.74 . \mathrm{C}_{22} \mathrm{H}_{18} \mathrm{Cl}_{2} \mathrm{~N}_{6} \mathrm{O}_{4}$ calcd. for: $\mathrm{C}, 52.71 ; \mathrm{H}, 3.62 ; \mathrm{N}, 16.76 \%$ ); $v_{\max } 1739$ (sydnone $\mathrm{C}=\mathrm{O}), 1613(\mathrm{C}=\mathrm{N}) ; \delta_{\mathrm{H}}\left(\mathrm{DMSO}-\mathrm{d}_{6}\right) 6.41(2 \mathrm{H}, \mathrm{d}, \mathrm{Ar}-\mathrm{H}), 7.16$ $(2 \mathrm{H}, \mathrm{d}, \mathrm{Ar}-\mathrm{H}), 7.5(2 \mathrm{H}, \mathrm{s}, \mathrm{Ar}-\mathrm{H}), 3.87\left(6 \mathrm{H}, \mathrm{s}, 2 \mathrm{CH}_{3}\right), 2.0$ $\left(6 \mathrm{H}, \mathrm{s}, 2 \mathrm{CH}_{3}\right) ; \mathrm{m} / \mathrm{z} 501\left(\mathrm{M}^{+}\right) ; 3$ n $(70 \%)$, m.p. 213-214 (Found : $\mathrm{C}, 49.53 ; \mathrm{H}, 3.38 ; \mathrm{N}, 15.75 . \mathrm{C}_{22} \mathrm{H}_{18} \mathrm{Cl}_{2} \mathrm{~N}_{6} \mathrm{O}_{6}$ calcd. for: $\mathrm{C}, 49.55 ; \mathrm{H}, 3.40 ; \mathrm{N}, 15.76 \%$ ); $\mathrm{v}_{\max } 1745$ (sydnone $\mathrm{C}=\mathrm{O}), 1610(\mathrm{C}=\mathrm{N}) ; \delta_{\mathrm{H}}\left(\mathrm{DMSO}-d_{6}\right) 6.41(2 \mathrm{H}, \mathrm{d}, \mathrm{Ar}-\mathrm{H}), 7.16$ $(2 \mathrm{H}, \mathrm{d}, \mathrm{Ar}-\mathrm{H}), 7.5(2 \mathrm{H}, \mathrm{s}, \mathrm{Ar}-\mathrm{H}), 3.89\left(6 \mathrm{H}, \mathrm{s}, 2 \mathrm{CH}_{3}\right), 3.8$ $\left(6 \mathrm{H}, \mathrm{s}, 2 \mathrm{CH}_{3}\right) ; \mathrm{m} / \mathrm{z} 533\left(\mathrm{M}^{+}\right) ; 3 \mathrm{o}(75 \%)$, m.p. $208-209^{\circ}$ (Found : $\mathrm{C}, 47.13 ; \mathrm{H}, 2.32 ; \mathrm{N}, 16.47 . \mathrm{C}_{20} \mathrm{H}_{12} \mathrm{Cl}_{2} \mathrm{~F}_{2} \mathrm{~N}_{6} \mathrm{O}_{4}$ calcd. for : C, 47.17; H, 2.37; N, 16.50\%); $v_{\max } 1738$ (sydnone $\mathrm{C}=\mathrm{O}), 1613(\mathrm{C}=\mathrm{N}) ; \delta_{\mathrm{H}}\left(\mathrm{DMSO}-d_{6}\right) 6.48(2 \mathrm{H}, \mathrm{d}$, $\mathrm{Ar}-\mathrm{H}), 7.24(2 \mathrm{H}, \mathrm{d}, \mathrm{Ar}-\mathrm{H}), 7.6(2 \mathrm{H}, \mathrm{s}, \mathrm{Ar}-\mathrm{H}), 3.8(3 \mathrm{H}, \mathrm{s}$, $\left.\mathrm{CH}_{3}\right) ; m / 2509\left(\mathrm{M}^{+}\right)$.

Synthesis of $\mathrm{N}$-acetyl hydrazones of 4-acetyl-3-aryl sydnone (4a-o) : Hydrazones of 4-acetyl-3-arylsydnones $(0.002 \mathrm{~mol})$ were heated with acetic anhydride $(3 \mathrm{ml})$ on water-bath for $15 \mathrm{~min}$. The reaction mixture was cooled, poured into ice-water and the resultant solid separated was filtered. Bright yellow crystalline compounds were obtained after crystallisation from ethanol. 4a (76\%), m.p. 189-190 (Found : C, 55.35; H, 4.60; N, 21.50. $\mathrm{C}_{12} \mathrm{H}_{12} \mathrm{~N}_{4} \mathrm{O}_{3}$ calcd. for : C, 55.38; H, 4.65; N, 21.53\%); $v_{\max } 3300(\mathrm{NH}), 1755$ (sydnone $\mathrm{C}=\mathrm{O}$ ), 1656 (amide $\mathrm{C}=\mathrm{O}$ ); $\delta_{\mathrm{H}}\left(\mathrm{DMSO}-d_{6}\right) 10.20$ ( $1 \mathrm{H}$, br, exchanged with $\left.\mathrm{D}_{2} \mathrm{O}, \mathrm{NH}\right), 6.5-7.41(5 \mathrm{H}, \mathrm{m}, \mathrm{Ar}-$ $\mathrm{H}), 2.50\left(3 \mathrm{H}, \mathrm{s}, \mathrm{CH}_{3}\right), 2.0\left(3 \mathrm{H}, \mathrm{s}, \mathrm{CH}_{3}\right) ; \mathrm{m} / z 260\left(\mathrm{M}^{+}\right) ; 4 \mathbf{b}$ (80\%), m.p. $171-172^{\circ}$ (Found : C, 56.90; H, 5.12; N, 20.40. $\mathrm{C}_{13} \mathrm{H}_{14} \mathrm{~N}_{4} \mathrm{O}_{3}$ calcd. for : C, 56.93; H, 5.14; N, 20.43\%); $v_{\max } 3310(\mathrm{NH}), 1750$ (sydnone $\mathrm{C}=\mathrm{O}$ ), 1658 (amide $\mathrm{C}=\mathrm{O}$ ); $\delta_{\mathrm{H}}\left(\mathrm{DMSO}-d_{6}\right) 10.25\left(1 \mathrm{H}\right.$, br, exchanged with $\left.\mathrm{D}_{2} \mathrm{O}, \mathrm{NH}\right)$, $6.35(2 \mathrm{H}, \mathrm{d}, \mathrm{Ar}-\mathrm{H}), 6.95(2 \mathrm{H}, \mathrm{d}, \mathrm{Ar}-\mathrm{H}), 2.50\left(3 \mathrm{H}, \mathrm{s}, \mathrm{CH}_{3}\right)$, $2.0\left(3 \mathrm{H}, \mathrm{s}, \mathrm{CH}_{3}\right), 2.3\left(3 \mathrm{H}, \mathrm{s}, \mathrm{CH}_{3}\right) ; \mathrm{m} / \mathrm{z} 274\left(\mathrm{M}^{+}\right) ; 4 \mathrm{c}(60 \%)$, m.p. 121-122 (Found : C, 56.90; H, 5.11; N, 20.40 . $\mathrm{C}_{13} \mathrm{H}_{14} \mathrm{~N}_{4} \mathrm{O}_{3}$ calcd. for : C, 56.93; H, 5.14; N, 20.43\%); $v_{\max } 3305(\mathrm{NH}), 1752$ (sydnone $\mathrm{C}=\mathrm{O}$ ), 1650 (amide $\mathrm{C}=\mathrm{O}$ ); $\delta_{\mathrm{H}}\left(\mathrm{DMSO}-d_{6}\right) 10.25\left(1 \mathrm{H}, \mathrm{br}\right.$, exchanged with $\left.\mathrm{D}_{2} \mathrm{O}, \mathrm{NH}\right)$,
$6.40(1 \mathrm{H}, \mathrm{d}, \mathrm{Ar}-\mathrm{H}) .7 .20(1 \mathrm{H}, \mathrm{d}, \mathrm{Ar}-\mathrm{H}), 7.25(2 \mathrm{H}, \mathrm{m}, \mathrm{Ar}-\mathrm{H})$. $2.50\left(3 \mathrm{H}, \mathrm{s}, \mathrm{CH}_{3}\right), 2.1\left(3 \mathrm{H}, \mathrm{s}, \mathrm{CH}_{3}\right), 2.4\left(3 \mathrm{H}, \mathrm{s}, \mathrm{CH}_{3}\right) ; \mathrm{m} / \mathrm{z}$ $274\left(\mathrm{M}^{+}\right)$; 4d (68\%), m.p. 174-175 (Found : C, 53.75; H, 4.85; N, 19.28. $\mathrm{C}_{13} \mathrm{H}_{14} \mathrm{~N}_{4} \mathrm{O}_{4}$ calcd. for : C, 53.79; $\mathrm{H}, 4.86$; $\mathrm{N}, 19.30 \%$ ); $v_{\max } 3300(\mathrm{NH}), 1755$ (sydnone $\mathrm{C}=\mathrm{O}$ ), 1653 (amide $\mathrm{C}=\mathrm{O}) ; \delta_{\mathrm{H}}\left(\mathrm{DMSO}-d_{6}\right) 10.21$ ( $1 \mathrm{H}$, br. exchanged with $\left.\mathrm{D}_{2} \mathrm{O}, \mathrm{NH}\right), 6.40(2 \mathrm{H}, \mathrm{d}, \mathrm{Ar}-\mathrm{H}), 7.00(2 \mathrm{H}, \mathrm{d}, \mathrm{Ar}-\mathrm{H}), 2.50$ $\left(3 \mathrm{H}, \mathrm{s}, \mathrm{CH}_{3}\right), 2.0\left(3 \mathrm{H}, \mathrm{s}, \mathrm{CH}_{3}\right), 3.8\left(3 \mathrm{H}, \mathrm{s}, \mathrm{CH}_{3}\right) ; \mathrm{m} / \mathrm{z} .290$ $\left(\mathrm{M}^{+}\right) ; 4 \mathrm{e}(70 \%)$, m.p. $160-161^{\circ}$ (Found : C, 53.75; H, 4.85; $\mathrm{N}, 19.28 . \mathrm{C}_{13} \mathrm{H}_{14} \mathrm{~N}_{4} \mathrm{O}_{4}$ calcd. for : C, 53.79; H. 4.86; N, 19.30\%); $v_{\max } 3305(\mathrm{NH}) .1755$ (sydnone $\mathrm{C}=\mathrm{O}$ ), 1651 (amide $\mathrm{C}=\mathrm{O}) ; \delta_{\mathrm{H}}\left(\mathrm{DMSO}-d_{6}\right) 10.21(1 \mathrm{H}$, br, exchanged with $\left.\mathrm{D}_{2} \mathrm{O}, \mathrm{NH}\right), 6.45(2 \mathrm{H}, \mathrm{d}, \mathrm{Ar}-\mathrm{H}), 7.37(1 \mathrm{H}, \mathrm{d}, \mathrm{Ar}-\mathrm{H}), 7.85$ $(2 \mathrm{H}, \mathrm{m}, \mathrm{Ar}-\mathrm{H}), 2.50\left(3 \mathrm{H}, \mathrm{s}, \mathrm{CH}_{3}\right), 2.1\left(3 \mathrm{H}, \mathrm{s}, \mathrm{CH}_{3}\right), 3.8(3 \mathrm{H}$, s, $\left.\mathrm{CH}_{3}\right) ; \mathrm{m} / \mathrm{z} 290\left(\mathrm{M}^{+}\right) ; 4 \mathrm{f}(82 \%)$, m.p. $142-143^{\circ}$ (Found : $\mathrm{C}, 48.90 ; \mathrm{H}, 3.74 ; \mathrm{N}, 19.00 . \mathrm{C}_{12} \mathrm{H}_{11} \mathrm{ClN}_{4} \mathrm{O}_{3}$ calcd. for : $\mathrm{C}$, 48.91; H, 3.76; N, 19.01\%); $v_{\max } 3300(\mathrm{NH}), 1757(\mathrm{C}=\mathrm{O})$, $1653(\mathrm{C}=\mathrm{O}) ; \delta_{\mathrm{H}}\left(\mathrm{DMSO}-d_{6}\right) 10.20(1 \mathrm{H}$, br, exchanged with $\left.\mathrm{D}_{2} \mathrm{O}, \mathrm{NH}\right), 6.40(2 \mathrm{H}, \mathrm{d}, \mathrm{Ar}-\mathrm{H}), 7.00(2 \mathrm{H} . \mathrm{d}, \mathrm{Ar}-\mathrm{H}), 2.50$ $\left(3 \mathrm{H}, \mathrm{s}, \mathrm{CH}_{3}\right), 2.0\left(3 \mathrm{H}, \mathrm{s}, \mathrm{CH}_{3}\right) ; n / z, 294\left(\mathrm{M}^{+}\right): 4 \mathrm{~g}(86 \%)$, m.p. $157-158^{\circ}$ (Found : C, 48.90; H, 3.74; N, 19.00 . $\mathrm{C}_{12} \mathrm{H}_{11} \mathrm{ClN}_{4} \mathrm{O}_{3}$ calcd. for : $\left.\mathrm{C}, 48.91 ; \mathrm{H}, 3.76 ; \mathrm{N}, 19.01 \%\right)$; $v_{\max } 3305(\mathrm{NH}), 1755$ (sydnone $\left.\mathrm{C}=\mathrm{O}\right), 1651$ (amide $\mathrm{C}=\mathrm{O}$ ); $\delta_{\mathrm{H}}\left(\mathrm{DMSO}-d_{6}\right) 10.25(1 \mathrm{H}, \mathrm{br}, \mathrm{NH}), 6.41(1 \mathrm{H}, \mathrm{d}, \mathrm{Ar}-\mathrm{H}), 7.30$ $(1 \mathrm{H}, \mathrm{d}, \mathrm{Ar}-\mathrm{H}), 7.25(1 \mathrm{H}, \mathrm{m}, \mathrm{Ar}-\mathrm{H}), 7.8(1 \mathrm{H}, \mathrm{s}, \mathrm{Ar}-\mathrm{H}), 2.50$ $\left(3 \mathrm{H}, \mathrm{s}, \mathrm{CH}_{3}\right), 2.1\left(3 \mathrm{H}, \mathrm{s}, \mathrm{CH}_{3}\right) ; \mathrm{m} / z 294\left(\mathrm{M}^{+}\right) ; 4$ h $(85 \%)$, m.p. 106-107 (Found: C, 42.47; H, 3.22; N, 16.50 . $\mathrm{C}_{12} \mathrm{H}_{11} \mathrm{BrN}_{4} \mathrm{O}_{3}$ calcd. for : $\mathrm{C}, 42.50 ; \mathrm{H}, 3.27 ; \mathrm{N}, 16.52 \%$ ); $v_{\max } 3308(\mathrm{NH}), 1758$ (sydnone $\mathrm{C}=\mathrm{O}$ ), 1652 (amide $\mathrm{C}=\mathrm{O}$ ); $\delta_{\mathrm{H}}\left(\mathrm{DMSO}-d_{6}\right) 10.43\left(1 \mathrm{H}\right.$, br, exchanged with $\left.\mathrm{D}_{2} \mathrm{O}, \mathrm{NH}\right)$, $6.43(2 \mathrm{H}, \mathrm{d}, \mathrm{Ar}-\mathrm{H}), 7.32(2 \mathrm{H}, \mathrm{d}, \mathrm{Ar}-\mathrm{H}), 2.50\left(3 \mathrm{H}, \mathrm{s}, \mathrm{CH}_{3}\right)$, $2.2\left(3 \mathrm{H}, \mathrm{s}, \mathrm{CH}_{3}\right) ; \mathrm{m} / z 239\left(\mathrm{M}^{+}\right) ; \mathbf{4 i}(70 \%)$, m.p. 113-114 (Found : C, 52.80; H, 4.40; N, 17.58. $\mathrm{C}_{14} \mathrm{H}_{14} \mathrm{~N}_{4} \mathrm{O}_{5}$ calcd. for: $\mathrm{C}, 52.83 ; \mathrm{H}, 4.43 ; \mathrm{N}, 17.60 \%) ; v_{\max }(\mathrm{NH}), 1757$ (sydnone $\mathrm{C}=\mathrm{O}), 1740($ ester $\mathrm{C}=\mathrm{O}), 1653($ amide $\mathrm{C}=\mathrm{O}) ; \delta_{\mathrm{H}}$ (DMSO- $\left.d_{6}\right) 10.42\left(1 \mathrm{H}, \mathrm{br}\right.$, exchanged with $\left.\mathrm{D}_{2} \mathrm{O}, \mathrm{NH}\right), 6.75$ (2H, d, Ar-H), $7.37(2 \mathrm{H}, \mathrm{d}, \mathrm{Ar}-\mathrm{H}), 2.49\left(3 \mathrm{H}, \mathrm{s}, \mathrm{CH}_{3}\right), 2.1$ $\left(3 \mathrm{H}, \mathrm{s}, \mathrm{CH}_{3}\right), 3.8\left(3 \mathrm{H}, \mathrm{s}, \mathrm{CH}_{3}\right) ; m / z 318\left(\mathrm{M}^{+}\right) ; 4 \mathrm{j}(74 \%)$, m.p. 117-118 (Found: C, 54.20; H, 4.81; N, 16.84 . $\mathrm{C}_{15} \mathrm{H}_{16} \mathrm{~N}_{4} \mathrm{O}_{5}$ calcd. for : $\mathrm{C}, 54.21 ; \mathrm{H}, 4.85 ; \mathrm{N}, 16.86 \%$ ); $v_{\max } 3300(\mathrm{NH}), 1752$ (sydnone $\left.\mathrm{C}=\mathrm{O}\right), 1745$ (ester $\mathrm{C}=\mathrm{O}$ ), 1653 (amide $\mathrm{C}=\mathrm{O}) ; \delta_{\mathrm{H}}\left(\mathrm{DMSO}-d_{6}\right) 10.45(1 \mathrm{H}$, br, exchanged with $\left.\mathrm{D}_{2} \mathrm{O}, \mathrm{NH}\right), 6.87(2 \mathrm{H}, \mathrm{d}, \mathrm{Ar}-\mathrm{H}), 7.45(2 \mathrm{H}, \mathrm{d}$, $\mathrm{Ar}-\mathrm{H}), 2.50\left(3 \mathrm{H}, \mathrm{s}, \mathrm{CH}_{3}\right), 2.1\left(3 \mathrm{H}, \mathrm{s}, \mathrm{CH}_{3}\right), 3.8\left(2 \mathrm{H}, \mathrm{q}, \mathrm{CH}_{2}\right)$, $1.8\left(3 \mathrm{H}, \mathrm{t}, \mathrm{CH}_{3}\right) ; \mathrm{m} / \mathrm{z} 332\left(\mathrm{M}^{+}\right) ; 4 \mathbf{k}(80 \%)$, m.p. $179-180^{\circ}$ (Found : C, 58.30; H, 5.57; N, 19.40. $\mathrm{C}_{14} \mathrm{H}_{16} \mathrm{~N}_{4} \mathrm{O}_{3}$ calcd. for: C, 58.32; H, 5.59; N, 19.43\%); $v_{\max } 3300(\mathrm{NH}), 1752$ (sydnone $\mathrm{C}=\mathrm{O}$ ), 1653 (amide $\mathrm{C}=\mathrm{O}$ ): $\delta_{\mathrm{H}}\left(\mathrm{DMSO}-d_{6}\right) 10.35$ ( $1 \mathrm{H}$, br, exchanged with $\left.\mathrm{D}_{2} \mathrm{O}, \mathrm{NH}\right), 6.45(1 \mathrm{H}, \mathrm{d}, \mathrm{Ar}-\mathrm{H}), 7.21$ $(1 \mathrm{H}, \mathrm{d}, \mathrm{Ar}-\mathrm{H}), 7.5$ (1 H, s, Ar-H). $2.4\left(3 \mathrm{H}, \mathrm{s} . \mathrm{CH}_{3}\right), 2.1(3 \mathrm{H}$, 
$\left.\mathrm{s}, \mathrm{CH}_{3}\right), 2.0\left(3 \mathrm{H}, \mathrm{s}, \mathrm{CH}_{3}\right), 1.9\left(3 \mathrm{H}, \mathrm{s}, \mathrm{CH}_{3}\right) ; \mathrm{m} / 2288\left(\mathrm{M}^{+}\right)$; 41 (70\%), m.p.153-154 (Found: C, 58.30; H, 5.57; N, 19.40. $\mathrm{C}_{14} \mathrm{H}_{16} \mathrm{~N}_{4} \mathrm{O}_{3}$ calcd. for: $\mathrm{C}, 58.32 ; \mathrm{H}, 5.59 ; \mathrm{N}$, 19.43\%); $v_{\max } 3315(\mathrm{NH}), 1750$ (sydnone $\mathrm{C}=\mathrm{O}$ ), 1656 (amide $\mathrm{C}=\mathrm{O}) ; \delta_{\mathrm{H}}\left(\mathrm{DMSO}-d_{6}\right) 10.41(1 \mathrm{H}, \mathrm{br}$, exchanged with $\left.\mathrm{D}_{2} \mathrm{O}, \mathrm{NH}\right), 6.43(1 \mathrm{H}, \mathrm{d}, \mathrm{Ar}-\mathrm{H}), 7.18(1 \mathrm{H}, \mathrm{d}, \mathrm{Ar}-\mathrm{H}), 7.4(1 \mathrm{H}$, $\mathrm{s}, \mathrm{Ar}-\mathrm{H}), 2.50\left(3 \mathrm{H}, \mathrm{s}, \mathrm{CH}_{3}\right), 2.1\left(3 \mathrm{H}, \mathrm{s}, \mathrm{CH}_{3}\right), 2.0(3 \mathrm{H}, \mathrm{s}$, $\left.\mathrm{CH}_{3}\right), 1.9\left(3 \mathrm{H}, \mathrm{s}, \mathrm{CH}_{3}\right) ; m / z 288\left(\mathrm{M}^{+}\right) ; 4 \mathrm{~m}(72 \%)$, m.p. 151$152^{\circ}$ (Found : C, 50.55; H, 4.20; N, 18.10. $\mathrm{C}_{13} \mathrm{H}_{13} \mathrm{ClN}_{4} \mathrm{O}_{3}$ calcd. for : C. 50.58; H, 4.24; N, 18.15\%); $v_{\max } 3302(\mathrm{NH})$, 1755 (sydnone $\mathrm{C}=\mathrm{O}$ ), 1660 (amide $\mathrm{C}=\mathrm{O}$ ); $\delta_{\mathrm{H}}\left(\mathrm{DMSO}-d_{6}\right.$ ) $10.43\left(1 \mathrm{H}\right.$, br, exchanged with $\left.\mathrm{D}_{2} \mathrm{O}, \mathrm{NH}\right), 6.41(1 \mathrm{H}, \mathrm{d}, \mathrm{Ar}-$ $\mathrm{H}), 7.16(1 \mathrm{H}, \mathrm{d}, \mathrm{Ar}-\mathrm{H}), 7.5(1 \mathrm{H}, \mathrm{s}, \mathrm{Ar}-\mathrm{H}), 2.50\left(3 \mathrm{H}, \mathrm{s}, \mathrm{CH}_{3}\right)$, $2.1\left(3 \mathrm{H}, \mathrm{s}, \mathrm{CH}_{3}\right), 2.0\left(3 \mathrm{H}, \mathrm{s}, \mathrm{CH}_{3}\right) ; \mathrm{m} / z 308\left(\mathrm{M}^{+}\right) ; \mathbf{4 n}(60 \%)$, m.p. 147-148 (Found : C, 48.05; H, 4.01; N, 17.22. $\mathrm{C}_{13} \mathrm{H}_{13} \mathrm{ClN}_{4} \mathrm{O}_{4}$ calcd. for : C, 48.08; $\mathrm{H}, 4.04 ; \mathrm{N}, 17.25 \%$ ); $v_{\max } 3312(\mathrm{NH}), 1754$ (sydnone $\mathrm{C}=\mathrm{O}$ ), 1655 (amide $\mathrm{C}=\mathrm{O}$ ); $\delta_{\mathrm{H}}\left(\mathrm{DMSO}-d_{6}\right) 10.43\left(1 \mathrm{H}, \mathrm{br}\right.$, exchanged with $\left.\mathrm{D}_{2} \mathrm{O}, \mathrm{NH}\right)$, $6.41(1 \mathrm{H}, \mathrm{d}, \mathrm{Ar}-\mathrm{H}), 7.16(1 \mathrm{H}, \mathrm{d}, \mathrm{Ar}-\mathrm{H}), 7.5(1 \mathrm{H}, \mathrm{s}, \mathrm{Ar}-\mathrm{H})$, $2.50\left(3 \mathrm{H}, \mathrm{s}, \mathrm{CH}_{3}\right), 2.1\left(3 \mathrm{H}, \mathrm{s}, \mathrm{CH}_{3}\right), 3.8\left(3 \mathrm{H}, \mathrm{s}, \mathrm{CH}_{3}\right) ; \mathrm{m} / \mathrm{z}$ $324\left(\mathrm{M}^{+}\right)$; $40(70 \%)$, m.p. 181-183 (Found : C, 46.06; H, 3.20; $\mathrm{N}, 17.90 . \mathrm{C}_{12} \mathrm{H}_{10} \mathrm{ClFN}_{4} \mathrm{O}_{3}$ calcd. for : C, 46.09; $\mathrm{H}$, 3.22; N, 17.92\%); $v_{\max } 3375(\mathrm{NH}), 1758$ (sydnone $\mathrm{C}=\mathrm{O}$ ),
1652 (amide $\mathrm{C}=\mathrm{O}) ; \delta_{\mathrm{H}}\left(\mathrm{DMSO}-d_{6}\right) 10.45(1 \mathrm{H}$, br, exchanged with $\left.\mathrm{D}_{2} \mathrm{O}, \mathrm{NH}\right) .6 .48(1 \mathrm{H}, \mathrm{d}, \mathrm{Ar}-\mathrm{H}), 7.24(1 \mathrm{H}, \mathrm{d}$, $\mathrm{Ar}-\mathrm{H}), 7.6(1 \mathrm{H}, \mathrm{s}, \mathrm{Ar}-\mathrm{H}), 2.50\left(3 \mathrm{H}, \mathrm{s}, \mathrm{CH}_{3}\right), 2.1\left(3 \mathrm{H}, \mathrm{s}, \mathrm{CH}_{3}\right)$, $m / z, 312\left(\mathrm{M}^{+}\right)$.

\section{Acknowledgement}

We thank Dr. G. S. Puranik, retired Professor of Organic Chemistry and former Chairman, Department of Chemistry, Karnatak University, Dharwad, for encouragement and suggestions. Authors also wish to thank SIF, Indian Institute of Science, Bangalore and USIC Karnatak University, Dharwad, for providing spectral data.

\section{References}

1. B. G. Ugarkar, B. V. Badami and G. S. Puranik, Arch. Pharm. (Weinheim), 1978, 311, 109.

2. S. V. Badachihar and G. S. Puranik, Indian. J. Chem., Sect. B, 1985, 24, 228.

3. (a) Shanta G. Mullur and Bharati V. Badami, Indian J. Heterocyclic Chem., 1999, 8, 343; (b) J. R Kavali and B. V. Badami, Il Farmaco., 2000, 55, 406.

4. C. V. Greco, J. Tobias and L. B. Kier, J. Het. Chem., 1967, 4, 160 . 